\title{
Note on Lie Point Symmetries of Burgers Equations
}

I.L. FREIRE ${ }^{1}$, Centro de Matemática, Computação e Cognição, CMCC, UFABC, 09.210-170 Santo André, SP, Brasil

${ }^{2}$ Instituto de Matemática, Estatística e Computação Científica, IMECC, UNICAMP, 13083-859 Campinas, SP, Brasil.

\begin{abstract}
In this note we study the Lie point symmetries of a class of evolution equations and obtain a group classification of these equations. We also identify the classical Lie algebras that the symmetry Lie algebras are isomorphic to.
\end{abstract}

Keywords. Evolution equations, Lie point symmetry, symmetry Lie algebras.

\section{Introduction}

This work corresponds to the talk [8] given during the XXXII CNMAC - XXXII Congresso Nacional de Matemática Aplicada e Computacional - September, 8-11 2009, Cuiabá, Brazil.

To begin with, let $\emptyset \neq M \subseteq \mathbb{R}^{n}, u: M \rightarrow \mathbb{R}$ be an open set and a smooth function, respectively, $x \in M$ and $k \in \mathbb{N}$. We use $\partial^{k} u$ to denote the jet bundle corresponding to all $k$ th partial derivatives of $u$ with respect to $x$. We simply denote $\partial^{1} u$ by $\partial u$.

A Lie point symmetry generator of a PDE $F=F\left(x, u, \partial u, \cdots, \partial^{m} u\right)=0$ of order $m$ is a vector field

$$
S=\xi^{i}(x, u) \frac{\partial}{\partial x^{i}}+\eta(x, u) \frac{\partial}{\partial u}
$$

on $M \times \mathbb{R}$ such that $S^{(m)} F=0$ when $F=0$ and

$$
S^{(m)}:=S+\eta_{i}^{(1)}(x, u, \partial u) \frac{\partial}{\partial u_{i}}+\cdots+\eta_{i_{1} \cdots i_{m}}^{(m)}\left(x, u, \partial u, \cdots, \partial^{m} u\right) \frac{\partial}{\partial u_{i_{1} \cdots i_{m}}},
$$

where

$$
\begin{aligned}
\eta_{i}^{(1)} & :=D_{i} \eta-\left(D_{i} \xi^{j}\right) u_{j}, \\
\eta_{i_{1} \cdots i_{j}}^{(j)} & :=D_{i_{j}} \eta_{i_{1} \cdots i_{j-1}}^{(j-1)}-\left(D_{i_{j}} \xi^{l}\right) u_{i_{1} \cdots i_{j-1} l}, 2 \leq j \leq m,
\end{aligned}
$$

\footnotetext{
${ }^{1}$ igor.freire@ufabc.edu.br

${ }^{2}$ igor@ime.unicamp.br
} 
and

$$
D_{i}:=\frac{\partial}{\partial x^{i}}+u_{i} \frac{\partial}{\partial u}+u_{i j} \frac{\partial}{\partial u_{j}}+\cdots+u_{i i_{1} \cdots i_{m}} \frac{\partial}{\partial u_{i_{1} \cdots i_{m}}}+\cdots
$$

is the total derivative operator, for more details, see [2, 9, 12]. Here the summation over the repeated indexes is understood.

Applications of this method in differential equations can be found in $[2,3,4,5$, $9,10,11,12,13,16,17]$. We shall not present more preliminaries concerning the Lie point symmetries of differential equations supposing that the reader is familiar with the basic notions and methods of group analysis [2, 9, 12].

In [10], Lagno and Samoilenko studied the group classification of quasilinear equation

$$
u_{t}=F\left(x, t, u, u_{x}\right) u_{x x}+G\left(x, t, u, u_{x}\right),
$$

where $u=u(x, t)$, for general smooth functions $F$ and $G$.

When $F=1$ and $G=-u u_{x}$, Eq. (1.2) is communly known as Burgers equation because it was first studied by Burgers in the last century. In this work we shall call Burgers equation the general Eq. (1.2) when $F=\nu=$ const. and $G=-g(u) u_{x}$, where $g(u)$ is a smooth function.

In [13], the projectable Lie point symmetries of (1.2) was carried out with $F=0$ and $G=-g(u) u_{x}$, for particular choices of the function $g$. The results obained in [13] were generalized in [11]. For more details, see [13, 11].

The purpose of the present note is to illuminate the properties of the Burgers equation

$$
\nu u_{x x}=u_{t}+g(u) u_{x}
$$

from the point of view of the S. Lie Symmetry Theory.

The linear case $g(u)=k=$ const. shall not be considered here because we are interested in nonlinear cases. The particular case $g(u)=0$ can be found in $[2,12]$.

Regarding Burgers equation

$$
u_{x x}=u_{t}+u u_{x}
$$

see $[2,12]$.

For a discussion of group classification of diffusion-convection equations and applications of Lie point symmetry theory in evolution equations, see [16].

The importance of group classification of differential equations was first emphasized by Ovsiannikov in 1950s-1960s, when he and his school began a systematic research program of successfully applying modern group analysis methods to wide range of physically important problems.

Following Olver ([12], p. 182), we recall that to perform a group classification on a differential equation involving a generic function $g$ consists of finding the Lie point symmetries of the given equation with arbitrary $g$, and, then, to determine all possible particular forms of $g$ for which the symmetry group can be enlarged.

It is worth observing that for problems which arise from physics, quite often there exists a physical motivation for considering such specific cases. For example, see $[3,4,11,13,16,17]$.

The remaining of the paper is organized as follows. In section 2 we carry out the complete group classification of Burgers equation and in the section 3 we identify the classical Lie algebras that the symmetry Lie algebras are isomorphic to. 


\section{Main Result}

Let us consider Eq. (1.3) with $\nu \neq 0$ and $g^{\prime}(u) \neq 0$. In the remaining of this paper, we shall suppose that all functions are smooth and they are well defined.

Lemma 2.1. Let

$$
S=\xi(x, t, u) \frac{\partial}{\partial x}+\phi(x, t, u) \frac{\partial}{\partial t}+\eta(x, t, u) \frac{\partial}{\partial u}
$$

be a symmetry of Eq. (1.3). Then $\xi=\xi(x, t), \phi=\phi(t)$ and $\eta=\alpha(x, t) u+\beta(x, t)$.

Proof. From [1, 2], we conclude that $\xi=\xi(x, t), \phi=\phi(x, t)$ and $\eta=\alpha(x, t) u+$ $\beta(x, t)$. From [10], $\phi=\phi(t)$.

Lemma 2.2. The linearly independet set of determining equations of Eq. (1.3) is:

$$
\begin{gathered}
\phi^{\prime}(t)=2 \xi_{x}, \\
u \alpha_{t}-\nu u \alpha_{x x}+u g(u) \alpha_{x}+\beta_{t}-\nu \beta_{x x}+g(u) \beta_{x}=0, \\
\xi_{t}+2 \nu \alpha_{x}-g(u) \xi_{x}-u g^{\prime}(u) \alpha-g^{\prime}(u) \beta=0 .
\end{gathered}
$$

Proof. It follows from the invariance condition $S^{(2)} F=0$ whenever

$$
F=\nu u_{x x}-u_{t}-g(u) u_{x}=0 .
$$

See also $[10,6,7,16]$.

\section{Remark 1:}

1. The number of determining equations is greater than that presented in Lemma 2.2, but some of them are equivalent to others. Thus, we have only listed the linearly independent set of equations in order to determine the symmetries.

2. We have used especific Mathematica packages in order to obtain the determining equations and solve them. See [6, 7].

Teorema 2.1. The widest Lie point symmetry group of Burgers Eq. (1.3), with an arbitrary $g(u)$, is determined by the operators

$$
X=\frac{\partial}{\partial x}, \quad T=\frac{\partial}{\partial t} .
$$

For some special choices of the function $g(u)$ it can be extended in the cases listed below. We shall write only the generators additional to (2.5).

1. If $g(u)=u$, then $B_{11}=t x \frac{\partial}{\partial x}+t^{2} \frac{\partial}{\partial t}+(x-t u) \frac{\partial}{\partial u}, B_{12}=t \frac{\partial}{\partial x}+\frac{\partial}{\partial u}$ and

$$
B_{13}=x \frac{\partial}{\partial x}+2 t \frac{\partial}{\partial t}-u \frac{\partial}{\partial u} \text {. }
$$

2. If $g(u)=u^{p}, p \neq 0,1$, then the additional generator is

$$
B_{2}=x \frac{\partial}{\partial x}+2 t \frac{\partial}{\partial t}-\frac{1}{p} u \frac{\partial}{\partial u} \text {. }
$$


3. If $g(u)=\log u$, then the additional generator is $B_{3}=t \frac{\partial}{\partial x}+u \frac{\partial}{\partial u}$.

4. If $g(u)=e^{b u}, b=$ const. $\neq 0$, then $B_{4}=x \frac{\partial}{\partial x}+2 t \frac{\partial}{\partial t}-\frac{1}{b} \frac{\partial}{\partial u}$.

5. If $g(u)=\frac{1-u}{1+u}$, then $B_{5}=(x-t) \frac{\partial}{\partial x}+2 t \frac{\partial}{\partial t}+(1+u) \frac{\partial}{\partial u}$.

6. If $g(u)=\frac{1}{1+u}$, then $B_{6}=x \frac{\partial}{\partial x}+2 t \frac{\partial}{\partial t}+(1+u) \frac{\partial}{\partial u}$.

7. If $g(u)=\frac{u}{1+u}$, then the additional generator is

$$
B_{7}=(x+t) \frac{\partial}{\partial x}+2 t \frac{\partial}{\partial t}+(1+u) \frac{\partial}{\partial u} .
$$

8. If $g(u)=\frac{u}{1-u}$, then the additional generator is

$$
B_{8}=(x-t) \frac{\partial}{\partial x}+2 t \frac{\partial}{\partial t}+(u-1) \frac{\partial}{\partial u} \text {. }
$$

9. If $g(u)=\frac{1+u}{u}$, then the additional generator is

$$
B_{9}=(x+t) \frac{\partial}{\partial x}+2 t \frac{\partial}{\partial t}+u \frac{\partial}{\partial u} .
$$

Proof. If $g$ is an arbitrary function, in order to equations (2.3) and (2.4) be true, we must necessarily have $\xi_{t}+2 \nu \alpha_{x}=\xi_{x}=\alpha=\beta=0$. From equations (2.2) and (2.4) we conclude that $\xi=c_{1}=$ const. and $\phi=c_{2}=$ const.. Thus, the symmetry (2.1) is spanned by translations in $x$ and $t$.

The proof of the case $g(u)=u$ can be found in [2,12]. For the other cases, substituting the functions listed in the Theorem in equations (2.3) and (2.4), we obtain two identities in terms of $u, g(u), u g(u), g^{\prime}(u)$ and $u g^{\prime}(u)$. After solving it (or using $[6,7]$ ), we obtain the coefficients $\xi, \phi, \alpha$ and $\beta$ of symmetry (2.1).

\section{Remark 2:}

1. The arbitrary case is easy to understand. Since Eq. (1.3) does not have coefficients depending on $x$ and $t$, the translational invariance is immediate.

2. We emphasize that the cases $g(u)=u^{p}$ (including $p=1$ ), $g(u)=e^{b u}$ (with $b \neq 0)$ and $g(u)=\log u$ are known, see [16], for example.

3. To the best of our knowledge, the additional cases 5 to 9 are not previously listed in any paper.

\section{Symmetry Lie Algebras}

In this section we are interested in classifing the symmetry Lie algebras of Eq. (1.3). In the next theorem we only present the non-null Lie brackets. 
Teorema 3.1. The symmetry Lie algebras of the Burgers equations are as follows.

1. If $g(u)=u$, then $\left[X, B_{11}\right]=B_{12}, \quad\left[X, B_{13}\right]=X,\left[T, B_{11}\right]=B_{13}$,

$\left[T, B_{12}\right]=X,\left[X, B_{13}\right]=2 T, \quad\left[B_{11}, B_{13}\right]=-2 B_{11}, \quad\left[B_{12}, B_{13}\right]=-B_{12}$.

2. If $g(u)=u^{p}, p \neq 0,1$, then $\left[X, B_{2}\right]=X,\left[T, B_{2}\right]=2 T$.

3. If $g(u)=\log u$, then $\left[T, B_{3}\right]=X$.

4. If $g(u)=e^{b u}, b=$ const., then $\left[X, B_{4}\right]=X, \quad\left[T, B_{4}\right]=2 T$.

5. If $g(u)=\frac{1-u}{1+u}$, then $\left[X, B_{5}\right]=X,\left[T, B_{5}\right]=-X+2 T$.

6. If $g(u)=\frac{1}{1+u}$, then $\left[X, B_{6}\right]=X,\left[T, B_{6}\right]=2 T$.

7. If $g(u)=\frac{u}{1+u}$, then $\left[X, B_{7}\right]=X,\left[T, B_{7}\right]=X+2 T$.

8. If $g(u)=\frac{u}{1-u}$, then $\left[X, B_{8}\right]=X,\left[T, B_{7}\right]=-X+2 T$.

9. If $g(u)=\frac{1+u}{u}$, then $\left[X, B_{9}\right]=X,\left[T, B_{7}\right]=X+2 T$.

Let $\mathfrak{g}_{1}:=\left\{X, T, B_{11}, B_{12}, B_{13}\right\}$ and $\mathfrak{g}_{i}:=\left\{X, T, B_{i}\right\}, 2 \leq i \leq 9$.

It is immediate that $\mathfrak{g}_{2} \cong \mathfrak{g}_{4} \cong \mathfrak{g}_{6}, \mathfrak{g}_{5} \cong \mathfrak{g}_{8}, \mathfrak{g}_{7} \cong \mathfrak{g}_{9}$ and that, under the change $X \mapsto-X, \mathfrak{g}_{5} \cong \mathfrak{g}_{7}$.

Teorema 3.2. $\mathfrak{g}_{2} \cong \mathfrak{g}_{7}$.

Proof. Let $e_{1}:=X, e_{2}:=X+T$ and $e_{3}:=B_{7}$. Then,

$$
\left[e_{1}, e_{3}\right]=e_{1} \text { and }\left[e_{2}, e_{3}\right]=2 e_{2} \text {. }
$$

Following the notations employed in $[14,15]$, i.e., $A_{i, j}^{\alpha}$ means the $j$ th algebra of dimension $i$ and the superscript, if any, gives the value of the continuous parameter on which the algebra depends (for more details, see [14, 15]), the following result is a consequence from Theorems $3.1,3.2$ and the changes $e_{1}^{\prime}=e_{2}, e_{2}^{\prime}=e_{1}, e_{3}^{\prime}=\frac{1}{2} e_{3}$ in $(3.1)$.

Teorema 3.3. $\mathfrak{g}_{1} \cong A_{5,40}, \mathfrak{g}_{2} \cong \mathfrak{g}_{4} \cong \mathfrak{g}_{5} \cong \mathfrak{g}_{6} \cong \mathfrak{g}_{7} \cong \mathfrak{g}_{8} \cong \mathfrak{g}_{9} \cong A_{3,5}^{\frac{1}{2}}, \mathfrak{g}_{3} \cong A_{3,1}$. 


\section{Conclusions}

In this paper the group classification of Burgers equation (Eq. (1.3)) is performed. The main result is Theorem 2.1, where is presented the group classification of Eq. (1.3). Theorem 3.3 show us the classical Lie algebras that the symmetry Lie algebras are isomorphic to.

Some of the cases presented in Theorem 2.1 (1-4) are known. However, we do not aware of any paper containing the other cases, at least in the information sources available to us.

The most interesting fact concerning to the symmetry Lie algebras is, except to the classical Burgers equation and the log nonlinearity, all other cases have symmetry Lie algebras isomorphic.

\section{Acknowledgments}

We wish to thank the Organizers of the XXXII CNMAC - XXXII Congresso Nacional de Matemática Aplicada e Computacional - September, 8-11 2009, Cuiabá, Brazil, for having given us the opportunity to talk on this subject as well as for their warm hospitality.

We are grateful to the referees for their useful suggestions.

We would like to thank Lab. EPIFISMA (Proj. FAPESP) for having given him the opportunity to use excellent computer facilities.

Resumo. Neste trabalho classificamos as simetrias de Lie de uma classe de equações de evolução semilineares. Também identificamos a quais álgebras de Lie clássicas as álgebras de Lie de simetrias são isomorfas.

\section{References}

[1] G.W. Bluman, Simplifying the form of Lie groups admitted by a given differential equation, J. Math. Anal. Appl., 145 (1990), 52-62.

[2] G.W. Bluman, S. Kumei, "Symmetries and differential equations", Applied Mathematical Sciences 81, Springer, 1989.

[3] Y.D. Bozhkov, Divergence symmetries of semilinear polyharmonic equations involving critical nonlinearities, J. Diff. Equ., 225 (2006), 666-684.

[4] Y.D. Bozhkov, I.L. Freire, Group Classification of Semilinear Kohn-Laplace Equations, Nonlinear Anal., 68 (2008), 2552-2568.

[5] Y.D. Bozhkov, I.L. Freire, Conservations laws for critical Kohn-Laplace equations on the Heisenberg Group, J. Nonlinear Math. Phys., 15 (2008), 35-47.

[6] S. Dimas, D. Tsoubelis, SYM: A new symmetry-finding package for Mathematica, in "10th International Conference in Modern Group Analysis", 64-70, 2005. 
[7] S. Dimas, D. Tsoubelis, A new heuristic algorithm for solving overdetermined systems of PDEs in Mathematica, in "6th International Conference in Nonlinear Mathematical Physics", 20-26, 2005.

[8] I.L. Freire, Note on Lie point symmetries of Burgers Equations, Anais do CNMAC, 2, (2009).

[9] N.H. Ibragimov, "Transformation groups applied to mathematical physics", Translated from the Russian Mathematics and its Applications (Soviet Series), D. Reidel Publishing Co., Dordrecht, 1985.

[10] V.I. Lagno, A.M. Samoilenko, Group classification of nonlinear evolution equations. I. Invariance under semisimple local transformation groups, Differ. Equ. 38 (2002), 384-391.

[11] M. Nadjafikhah, Lie symmetries of inviscid Burgers' equation, Adv. Appl. Clifford Alg., 19 (2009), 101-112.

[12] P.J. Olver, "Applications of Lie groups to differential equations", GMT 107, Springer, New York, 1986.

[13] A. Ouhadan, E.H. El Kinani, Lie symmetries of the equation $u_{t}(x, t)+$ $g(u) u_{x}(x, t)=0$, Adv. Appl. Clifford Alg., 17 (2007), 95-106.

[14] J. Patera, R.T. Sharp, P. Winternitz, H. Zassenhaus, Invariants of real low dimension Lie algebras, J. Math. Phys., 17 (1976), 986-994.

[15] J. Patera, P. Winternitz, Subalgebras of real three- and four-dimensional Lie algebras, J. Math. Phys., 18 (1977), 1449-1455.

[16] R.O. Popovych, N.M. Ivanova, New results on group classification of nonlinear diffusion-convection equations, J. Phys. A: Math. Gen., 37 (2004), 7547-7565.

[17] S.R. Svirshchevskii, Group classification and invariant solutions of nonlinear polyharmonic equations, Differ. Equ./Diff. Uravn., 29 (1993), 1538-1547. 